\title{
Transcervical videomediastino-thoracoscopy
}

\author{
Josep Belda-Sanchis ${ }^{1}$, Joan Carles Trujillo-Reyes ${ }^{2}$, Carme Obiols ${ }^{1}$, Elisabeth Martínez-Téllez ${ }^{2}$, Sergi \\ Call $^{1}$, Mireia Serra-Mitjans ${ }^{1}$, Mauro Guarino ${ }^{2}$, Ramón Rami-Porta ${ }^{1,3}$
}

${ }^{1}$ Department of Thoracic Surgery, Hospital Universitari Mútua Terrassa, University of Barcelona, Barcelona, Spain; ${ }^{2}$ Department of Thoracic Surgery, Hospital Universitari de la Santa Creu i Sant Pau, Autonomous University of Barcelona, Barcelona, Spain; ${ }^{3}$ Network of Centres for Biomedical Research in Respiratory Diseases (CIBERES) Lung Cancer Group, Barcelona, Spain

Correspondence to: Josep Belda-Sanchis, MD, PhD. Department of Thoracic Surgery, Hospital Universitari Mútua Terrassa, Plaza Dr. Robert, 5, 08221 Terrassa, Barcelona, Spain. Email: jbelda@mutuaterrassa.es.

\begin{abstract}
Although technical advances in non-invasive and minimally invasive approaches to lung and pleural cancer diagnosis and staging have become more widely available and accurate, surgical techniques remain the gold standard in assessing the extent of loco-regional involvement. Precise surgical staging of lung or pleural tumours is pivotal in the selection of surgical candidates and for predicting survival. In some patients, both mediastinal and pleural exploration may be needed for many different reasons. Transcervical videomediastino-thoracoscopy (VMT) combines simultaneously the exploration of both the mediastinum and the pleural cavities through a single cervical incision, allowing for biopsies or sampling of the mediastinal lymph nodes, lymphadenectomy and pleuropulmonary assessment (mainly pleural effusions, tumour involvement of the visceral and parietal pleura and pulmonary nodules). Thoracic surgeons should be aware of this combined surgical approach and completely familiar with classical indications and technical details of the transcervical approach to the mediastinum and thoracoscopic exploration of the pleural cavities.
\end{abstract}

Keywords: Staging; lung cancer; videomediastinoscopy; thoracoscopy

Submitted Feb 27, 2018. Accepted for publication Mar 13, 2018.

doi: $10.21037 /$ jtd.2018.03.132

View this article at: http://dx.doi.org/10.21037/jtd.2018.03.132

\section{Introduction}

Transcervical videomediastino-thoracoscopy (VMT) was described for the first time by Deslauriers et al. in 1976 to obtain pathological diagnosis in patients with bronchogenic carcinoma, benign pulmonary disease, and pleural disease. By the combination of simultaneous exploration of the mediastinum and pleura through a standard cervical mediastinoscopy incision, the pathological diagnosis was obtained in more than $80 \%$ of cases. The conclusion of their experience was that transcervical VMT is safe and a very useful alternative method to get tissue for definitive pathologic diagnosis of many pulmonary and pleural diseases (1).

The staging of lung and pleural cancers defines the anatomical extent of disease and its accuracy is pivotal for planning treatment and predicting survival. Staging from surgical explorations provides the highest clinical certainty.
Transcervical VMT combines through a single cervical incision two surgical explorations that are usually done separately by thoracic surgeons: a classical or extended videomediastinoscopy and a thoracoscopy. The classical videomediastinoscopy provides access to the upper and lower right and left paratracheal lymph nodes, as well as the subcarinal ones, allowing us to biopsy or completely remove [video-assisted mediastinoscopic lymphadenectomy (VAMLA)] the lymph nodes from these stations (2-6). The extended cervical videomediastinoscopy provides access to the aorto-pulmonary window lymph nodes (7). Thoracoscopy enables access to the chest cavities allowing us to take biopsies from the pleura, lung and mediastinal and hilar lymph nodes, as well as making some therapeutic or diagnostic manoeuvres such as pleurodesis for treatment of recurrent malignant pleural effusions, lung biopsies or lung nodules resection.

The main indications of the transcervical VMT would 


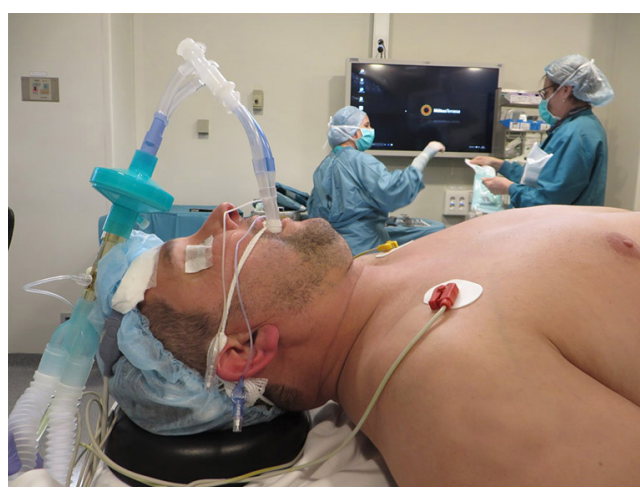

Figure 1 Patient's head hyperextended. A head ring provides stability during surgery.

be $(8,9)$ :

(I) The diagnosis and staging of lung cancer. Patients in which a surgical exploration of the mediastinum is indicated and who have an associated cytological negative pleural effusion. In these patients both, lymph node and pleural malignancy must be confirmed or ruled out before lung resection;

(II) The diagnosis and staging of malignant pleural mesothelioma. The transcervical VMT allows us to explore the mediastinum and both chest cavities;

(III) The pleurodesis for symptomatic control of malignant pleural effusions detected on any of the previous scenarios;

(IV) The resection of accessible pulmonary nodules (e.g., nodules located in upper lobes and middle lobe).

One of the transcervical VMT main advantages is that it would avoid the intercostal nerve injury and acute postoperative pain due to the thoracoscopy port or chest tube. Another potential advantage is a risk reduction of wound recurrence due to its contamination with tumour cells in patients with malignant pleural mesothelioma $(10,11)$.

Definitive or relative contraindications of the technique are mediastinal adhesions preventing safe entry to the pleural cavity or obliterated pleural spaces due to previous radiotherapy, surgery or infection, mediastinal pleura invasion (e.g., huge tumour on the mediastinal surface in mesothelioma or an upper lobe lung tumour invading the mediastinum), lung nodules located posteriorly (e.g., basal segments of the lower lobes), severe neck rigidity, large goitres, ascending aortic aneurism or atheromatosis of the aorta $(2,7,8,11,12)$.

The most frequent complication is the transient or permanent left recurrent laryngeal nerve palsy caused during the surgical exploration of the left paratracheal space or the aorto-pulmonary window. In our experience, after one hundred and 60 VAMLAs performed for non-small cell lung cancer staging, left recurrent laryngeal nerve palsy occurred in $3.2 \%$ of the patients (6). Visual identification of the nerve during dissection and avoidance of the electrocautery in close proximity to the nerve are the keys in reducing the risk of injury. Bleeding from small vessels near the recurrent nerve should be controlled by temporary compression or tamponade with a cotton or a gauze, or by carefully clipping. Other described complications include mediastinitis, wound infection, haemorrhage, prolonged air leak, residual pneumothorax and wound tumour recurrence $(10,13)$. In order to reduce the risk of incisional tumour seeding, all the procedures should be done through the working channel of the video mediastinoscope.

Another potential complication of the transcervical VMT is hemothorax. According to El-sayed, the pleurotomy could prevent the self-tamponade of the mild venous bleeder after the videomediastinoscopy, as the blood trickled down from the mediastinum into the chest cavity (14).

\section{Operative technique}

\section{Anaesthetic considerations and patient's position}

Under general anaesthesia, the patient is positioned in a supine position. Differently from the standard cervical videomediastinoscopy, a double-lumen endotracheal tube placement is mandatory to achieve single lung ventilation. Skin preparation, draping and patient's position are the same of a standard videomediastinoscopy $(2,7)$. Important points to keep in mind while positioning the patients are maximal hyperextension of the neck by placing a sandbag or cushion under the patient's shoulders to raise the chest and facilitate the range of movement of the mediastinoscope, and patient's head stabilization on a head ring (Figure 1).

\section{Instrumentation}

Both the Linder-Dahan spreadable blade video mediastinoscope (Richard Wolf, Knittlingen, Germany) and the Lerut DCI video mediastinoscope (KARL STORZ SE \& Co. KG, Tuttlingen, Germany) can be used. By opening the blades distally and in parallel, the Linder-Dahan video mediastinoscope provides a wider operative field. In addition, it can easily be fixed to a U-shaped support arm, thus facilitating the bimanual dissection (Figure 2). 


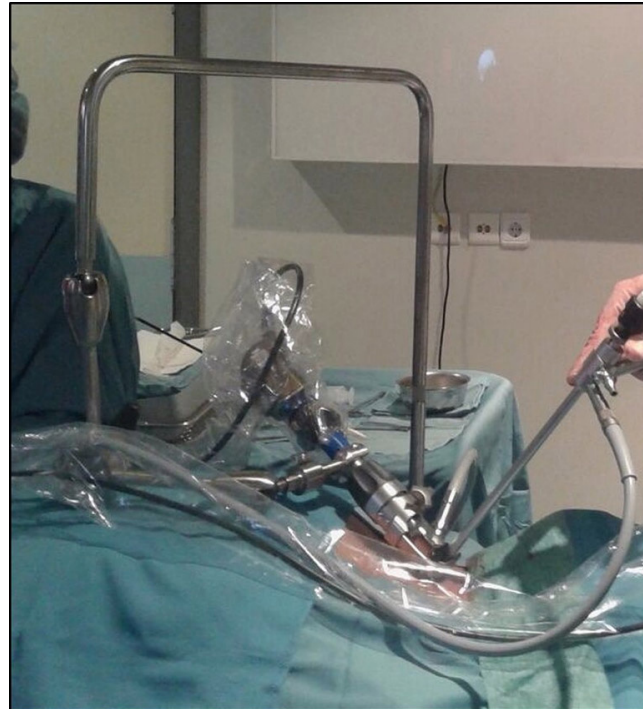

Figure $2 \mathrm{U}$-shaped retaining arm with special clamping device used to fix and stabilize the video mediastinoscope (Richard Wolf, Knittlingen, Germany).

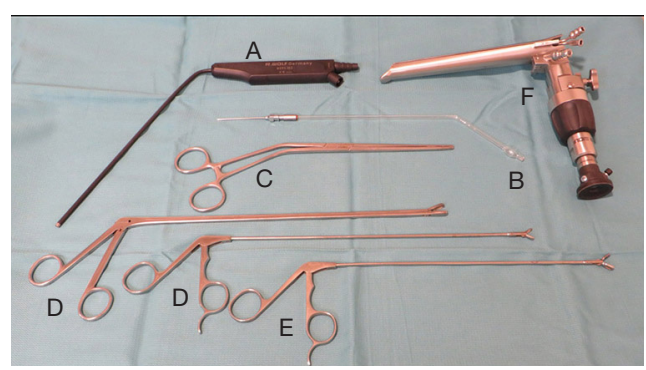

Figure 3 Set of specific instruments required for videomediastinoscopy: (A) dissection-suction-coagulation cannula; (B) glass tube for test puncture; (C) grasping forceps to retrieve inserted gauzes; (D) biopsy forceps; (E) grasper; (F) Linder-Dahan spreadable blade video mediastinoscope (Richard Wolf, Knittlingen, Germany).

A full set of specific instruments for videomediastinoscopy include: a dissection-suction-coagulation cannula, grasping forceps to retrieve inserted gauzes, endoscopic scissors, biopsy forceps and glass tube for test puncture (Figure 3).

A 5- or 10-mm, 0- or 30-degree angle videothoracoscope is used for inspecting the pleural cavity. A 30-degree viewing angle videothoracoscope is preferred because it provides a panoramic view of the whole chest cavity as well as of areas difficult to visualise by simply rotating the thoracoscope on its longitudinal axis. Alternatively, a bayonet thoracoscope with a working channel can be used (Figure 4).

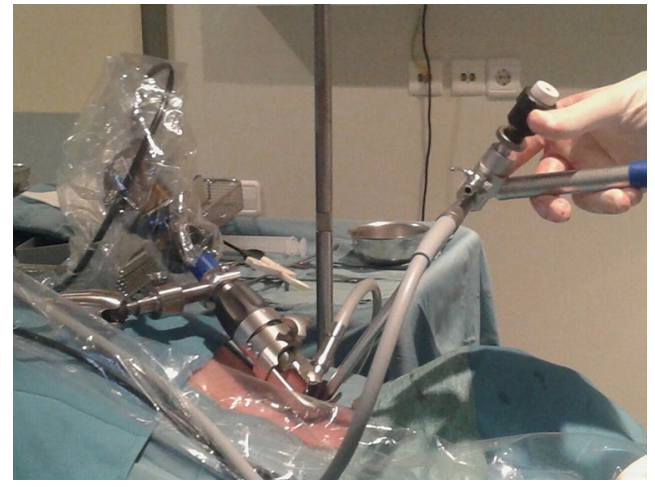

Figure 4 A bayonet thoracoscope with a working channel inserted through the video mediastinoscope.

The same suction cannula and forceps used for the videomediastinoscopy, can be used to remove the pleural fluid if present (this fluid should be sent for cytological and biochemical analyses) and to perform biopsies of the pleura for pathological examination.

The use of two monitors, one for the videomediastinoscope and another one for the thoracoscope, allows for a continuous view of the surgical field.

\section{Surgical procedure}

First, planned surgical exploration of the mediastinum is performed (lymph node biopsies, sampling or complete en bloc removal of the fatty tissue and lymph nodes from the subcarinal, right and left paratracheal, for right-sided cancers; the subaortic and para-aortic nodal stations are added for left-sided cancers by means of extended cervical videomediastinoscopy (nodal stations 7, 4R, 4L, 5 and 6, respectively).

If the pathological diagnosis is not established after the surgical exploration of the mediastinum by intra-operative frozen section evaluation, or a more exhaustive diagnostic or staging study of the tumour should be needed, then, the pleural cavity is entered by instrumental mediastinal pleurotomy.

On the right side, mediastinal pleurotomy is made under endoscopic view just caudal to the innominate artery. The superior vena cava may be pulled up or down to facilitate the exposition of the mediastinal pleura, which can be entered anteriorly or posteriorly to the vein (Figures 5-7).

On the left side, a tunnel is created by digital blunt dissection of the fascia between the innominate artery and 


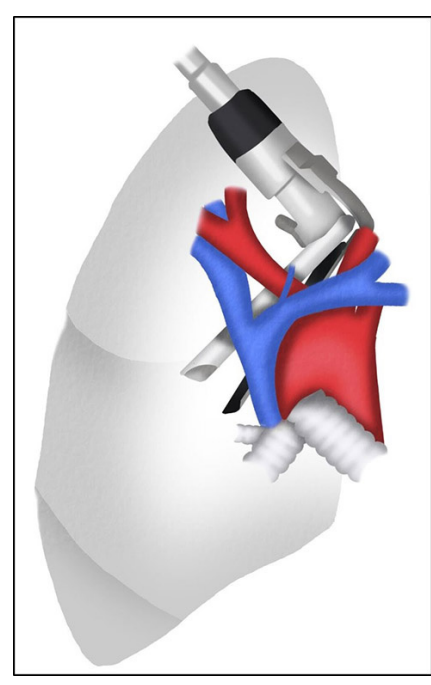

Figure 5 Transcervical access to the right pleural cavity.

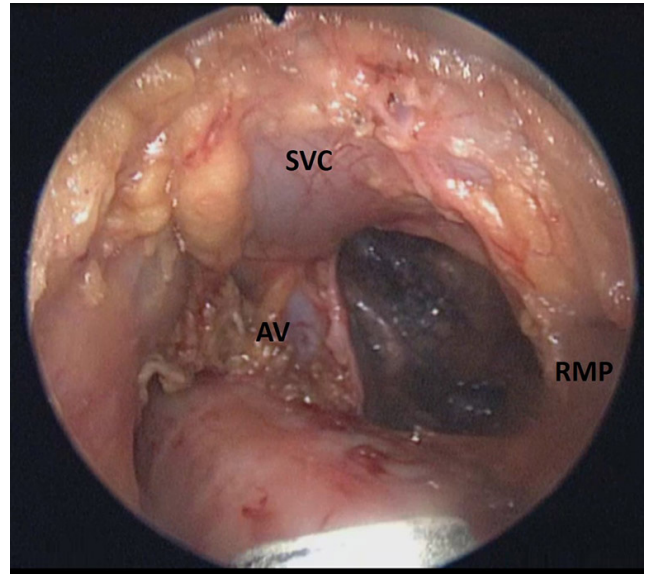

Figure 6 The SVC pulled up to facilitate the exposition of the RMP and pleurotomy. AV, azygos vein. AV, azygos vein; SVC, superior vena cava; RMP, right mediastinal pleura.
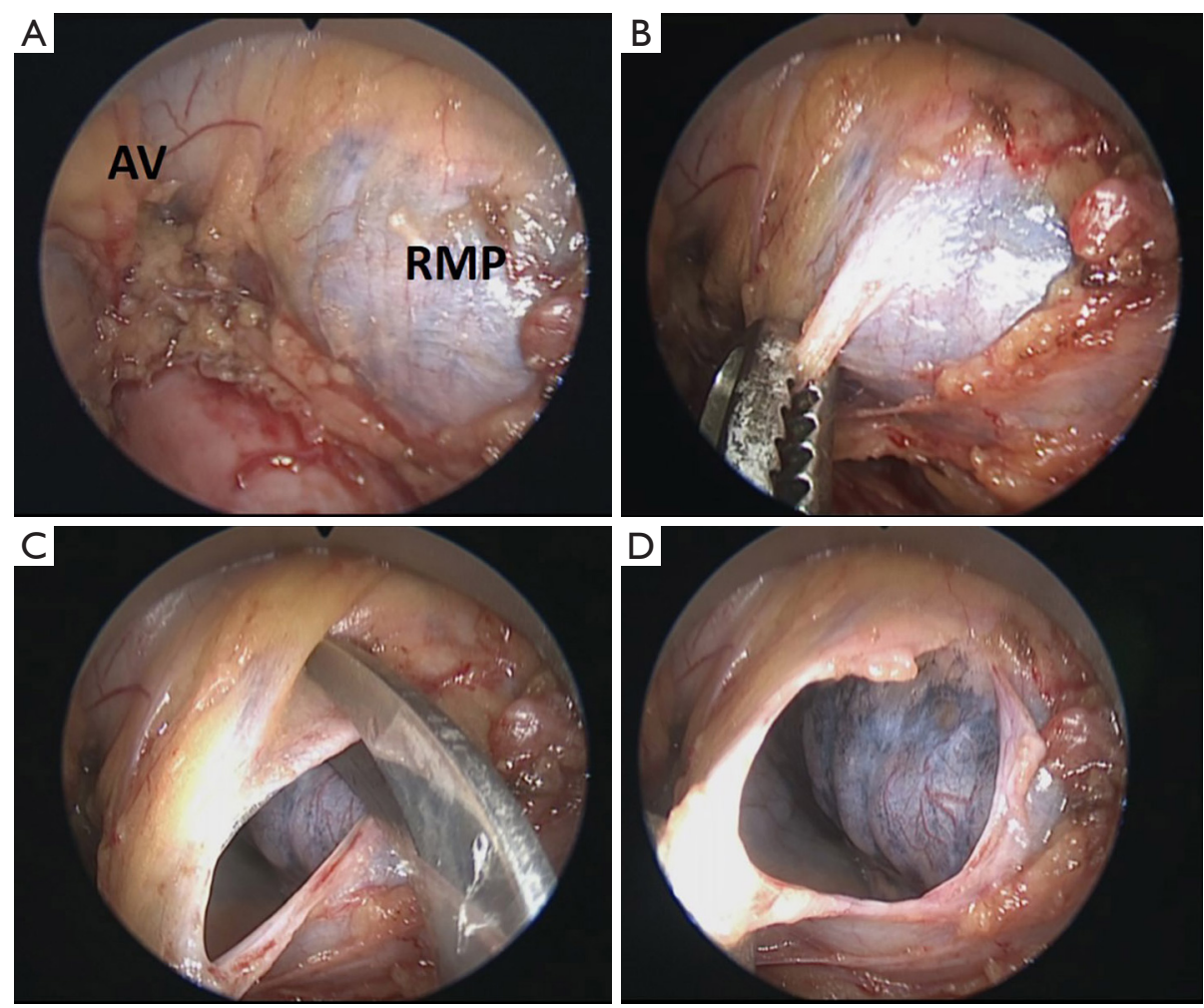

Figure 7 The sequence of figures shows the main steps of the right transcervical videomediastino-thoracoscopy. (A) RMP exposed; (B) by grasping and pulling the mediastinal pleura; (C,D) the pleurotomy can be performed using endoscopic scissors. AV, azygos vein; RMP, right mediastinal pleura. 


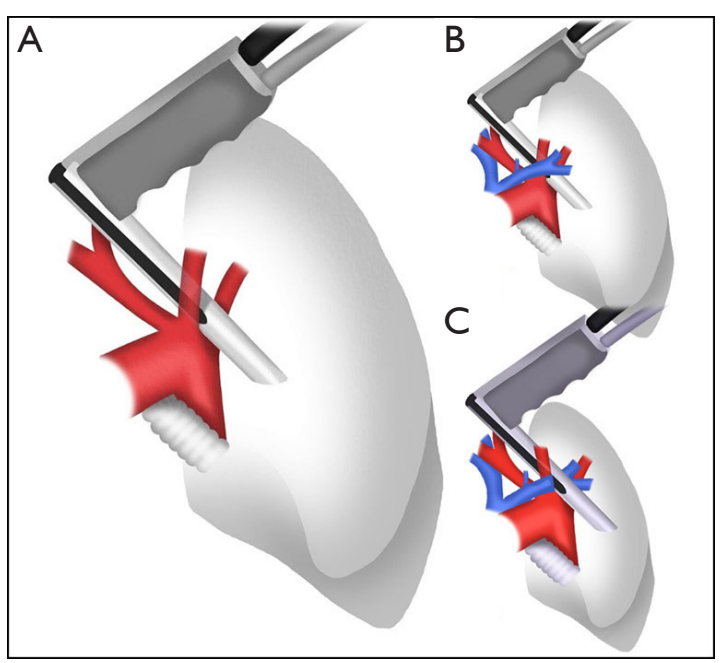

Figure 8 Video mediastinoscope inserted between the innominate artery and the left common carotid artery at their origin in the aortic arch (A) and advanced in front (B) or behind (C) the left innominate vein.

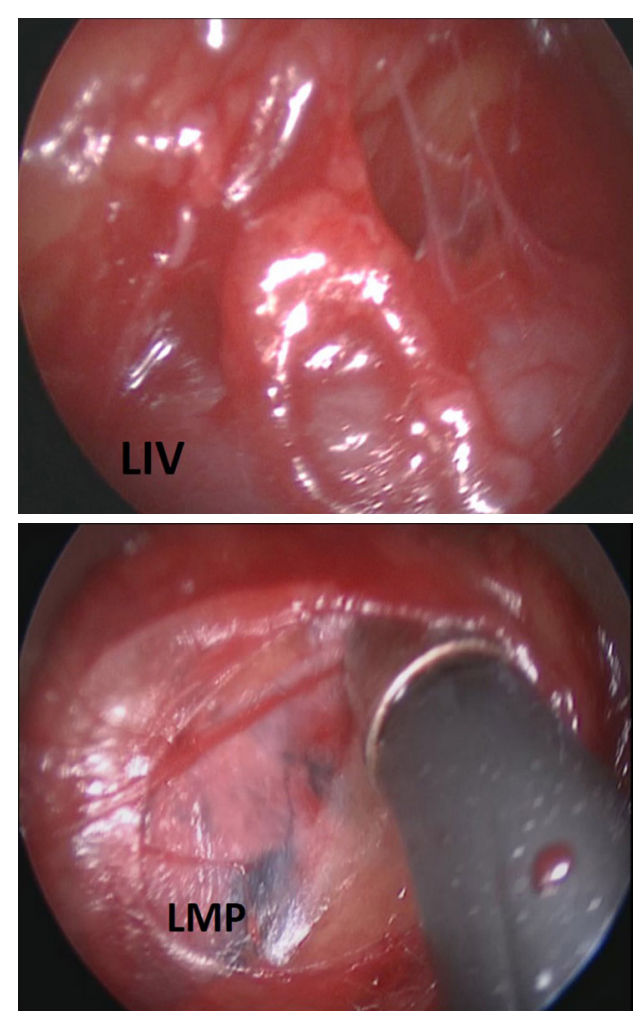

Figure 9 Transcervical access to the left pleural cavity in front of the LIV. Exposition of the LMP at the level of the aortopulmonary window. LIV, left innominate vein; LMP, left mediastinal pleura.

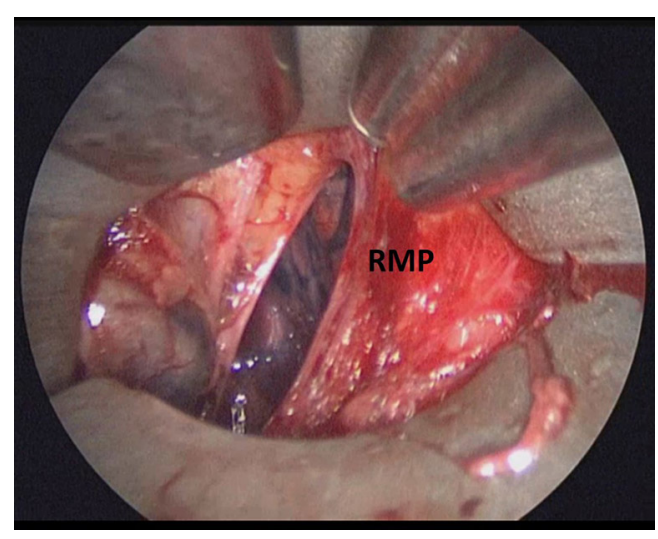

Figure 10 Thoracoscopic view of the right pleurotomy through the working channel of the video mediastinoscope. RMP, right mediastinal pleura.

the left common carotid artery at their origin in the aortic arch. The video mediastinoscope is then inserted and advanced in front or behind the left innominate vein until the aorto-pulmonary window and left mediastinal pleura is reached (15) (Figures 8,9). Then, the pleurotomy is made under endoscopic view and the left pleural cavity examined.

Remarkably, access to the right chest cavity tends to be less risky and much easier than the access to the left side. In order to reduce the risk of vascular or neurological lesion, Assouad et al. have described in an experimental cadaveric model a new approach to the left thoracic cavity through a cervical incision, passing through the retrosternal space. The authors used a flexible video endoscope through the working channel of the video mediastinoscope to reach and explore the left thoracic cavity and the aorto-pulmonary window (16-18).

The video mediastinoscope and/or the videothoracoscope are advanced into the pleural cavity through the pleurotomy (Figure 10). If necessary, the pleural fluid is removed with a suction catheter. Parietal pleural biopsies, lung biopsies, lung nodule resection and talc pleurodesis (when pleural carcinomatosis is present) can be performed.

After the procedure is completed, a catheter or chest tube is inserted through a separate stab wound or through the cervical incision itself to remove air from the chest cavity (Figures 11,12).

\section{Comments}

Accurately determining the diagnosis and stage of thoracic malignancies is crucial to treatment selection and in 


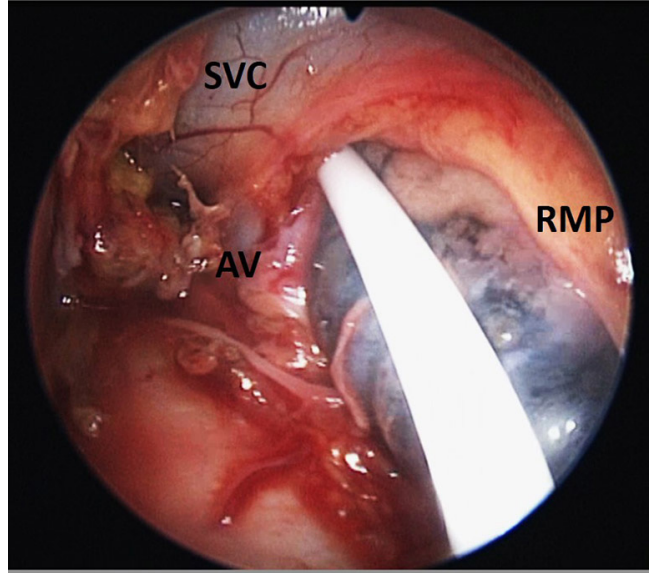

Figure 11 View of the small drainage tube introduced into the pleural cavity. SVC, superior vena cava; RMP, right mediastinal pleura; AV, azygos vein.

establishing the patient's prognosis, but the process is often complex and requires an efficient test protocol. Despite the great progress that has been made in less invasive techniques, surgical exploration of the mediastinum and pleural space still plays a central role in the protocol of diagnosis and staging of thoracic malignancies.

In some cases, both the mediastinum and the pleural cavity need to be explored to obtain a pathological diagnosis or a complete staging. VMT combines through a single cervical incision a videomediastinoscopy and a thoracoscopy, two surgical procedures that are usually done separately (19-23). Combined VMT preserves the advantages of both procedures, overcoming some of the drawbacks related to the use of intercostal ports and chest drains.

Although the literature on the VMT is scarce, it has been described as a safe, reproducible and valuable procedure for diagnosing and staging thoracic malignancies in selected patients. VMT can be a good alternative for simultaneous exploration of mediastinum, pleural space and lung parenchyma. In addition, non-complex therapeutic procedures such as evacuation of pleural liquid and talc pleurodesis or excision of pulmonary nodules can be performed using the working channels of the video mediastinoscope and thoracoscope $(1,8-12)$.

We believe that VMT should be considered as one more technique among the various procedures available for achieving a cyto-histologic diagnosis and for staging of thoracic malignancies, particularly when both mediastinum and the pleural cavities need to be explored. Training across all of the videomediastinoscopic and thoracoscopic
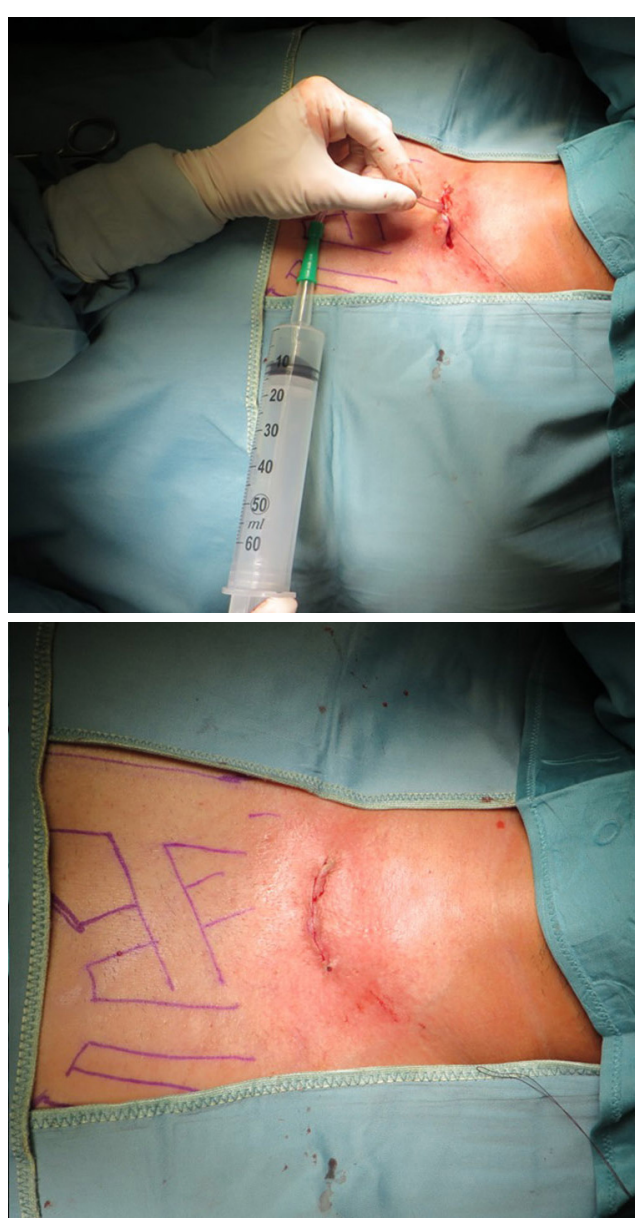

Figure 12 In this case, a nasogastric tube was introduced into the pleural cavity through the opened mediastinal pleura and exteriorized through the cervicotomy. A $50 \mathrm{~mL}$ syringe was connected directly to the nasogastric tube and constant negative pressure is applied to the plunger of the syringe to aspirate air from the chest cavity.

approaches is the key to maximize its safety and value.

\section{Acknowledgements}

We would like to express my gratitude and appreciation to Aitana Belda-Camós for drawing Figures 5 and 8.

\section{Footnote}

Conflicts of Interest: The authors have no conflicts of interest to declare.

Informed Consent: Written informed consent was obtained 
from the patient for publication of this paper and any accompanying images.

\section{References}

1. Deslauriers J, Beaulieu M, Dufour C, et al. Mediastinopleuroscopy: a new approach to the diagnosis of intrathoracic diseases. Ann Thorac Surg 1976;22:265-9.

2. Rami-Porta R, Call S, Serra-Mitjans M. Mediastinoscopy. In: Zieliński M, Rami-Porta R. editors. The Transcervical Approach in Thoracic Surgery. Heidelberg: SpringerVerlag, 2014:9-27.

3. Witte B, Wolf M, Hürtgen M. Video-assisted mediastinoscopic lymphadenectomy (VAMLA). In: Zieliński M, Rami-Porta R. editors. The Transcervical Approach in Thoracic Surgery. Heidelberg: Springer-Verlag, 2014:89-99.

4. Hürtgen M, Friedel G, Toomes H, et al. Radical video-assisted mediastinoscopic lymphadenectomy (VAMLA)--technique and first results. Eur J Cardiothorac Surg 2002;21:348-51.

5. Witte B, Hürtgen M. Video-assisted mediastinoscopic lymphadenectomy (VAMLA). J Thorac Oncol 2007;2:367-9.

6. Call S, Obiols C, Rami-Porta R, et al. Video-assisted mediastinoscopic lymphadenectomy for staging non-small cell lung cancer. Ann Thorac Surg 2016;101:1326-33.

7. Call S, Obiols C, Rami-Porta R. Extended cervical mediastinoscopy. In: Zieliński M, Rami-Porta R. editors. The Transcervical Approach in Thoracic Surgery. Heidelberg: Springer-Verlag, 2014:29-35.

8. Dawson AG, Waller DA. Cervical mediastinothoracoscopy. In: Zieliński M, Rami-Porta R. editors. The Transcervical Approach in Thoracic Surgery. Heidelberg: Springer-Verlag, 2014:45-51.

9. Obiols C, Call S, Rami-Porta R, et al. Utility of the transcervical approach in bilateral synchronous lung cancer. Asian Cardiovasc Thorac Ann 2015;23:991-4.

10. Chamberlain MH, Fareed K, Nakas A, et al. Videoassisted cervical thoracoscopy: a novel approach for diagnosis, staging and pleurodesis of malignant pleural mesothelioma. Eur J Cardiothorac Surg 2008;34:200-3.

11. Fowkes L, Lau KK, Shah N, et al. A cervical approach to investigating pleural disease. Ann Thorac Surg 2009;88:315-7.

12. Trujillo-Reyes JC, Martínez-Téllez E, Rami-Porta R, et al. Combination video-assisted mediastinoscopic lymphadenectomy and transcervical thoracoscopy. Multimed Man Cardiothorac Surg 2018. doi: 10.1510/ mmcts.2018.004.

13. Obiols C, Call S, Rami-Porta R, et al. Extended cervical mediastinoscopy: mature results of a clinical protocol for staging bronchogenic carcinoma of the left lung. Eur J Cardiothorac Surg 2012;41:1043-6.

14. Elsayed H. Haemothorax after mediastinoscopy: a word of caution. Eur J Cardiothorac Surg 2012;41:138-9.

15. Ginsberg RJ, Rice TW, Goldberg M, et al. Extended cervical mediastinoscopy. A single staging procedure for bronchogenic carcinoma of the left upper lobe. J Thorac Cardiovasc Surg 1987;94:673-8.

16. Assouad J, Fénane H, Masmoudi H, et al. Flexible endoscope in thoracic surgery: CITES or cVATS? Rev Pneumol Clin 2013;69:294-7.

17. Masmoudi H, Karsenti A, Giol M, et al. Cervical and retrosternal approach to the left thoracic cavity using a flexible endoscope. Interact Cardiovasc Thorac Surg 2014;18:784-8.

18. Assouad J, Steltzlen C, Masmoudi H, et al. Cervical incision thoracic endoscopic surgery: a minimally invasive endoscopic approach in thoracic surgery. Interact Cardiovasc Thorac Surg 2010;10:967-70.

19. Landreneau RJ, Hazelrigg SR, Mack MJ, et al. Thoracoscopic mediastinal lymph node sampling: useful for mediastinal lymph node stations inaccessible by cervical mediastinoscopy. J Thorac Cardiovasc Surg 1993;106:554-8.

20. Mouroux J, Venissac N, Alifano M. Combined videoassisted mediastinoscopy and video-assisted thoracoscopy in the management of lung cancer. Ann Thorac Surg 2001;72:1698-704.

21. Mouroux J, Venissac N, Alifano M. Combined videoassisted mediastinoscopy and thoracoscopy in the management of lung cancer: a five-year experience. J Laparoendosc Adv Surg Tech A 2005; 15:460-9.

22. Sawabata N. Combined video-assisted mediastinoscopy and video-assisted thoracoscopy in the staging of lung cancer. Kyobu Geka 2007;60:699-705.

23. Pinto Filho DR, Avino AJ, Brandão SL, et al. Joint use of cervical mediastinoscopy and video-assisted thoracoscopy for the evaluation of mediastinal lymph nodes in patients with non-small cell lung cancer. J Bras Pneumol 2009;35:1068-74.

Cite this article as: Belda-Sanchis J, Trujillo-Reyes JC, Obiols C, Martínez-Téllez E, Call S, Serra-Mitjans M, Guarino M, Rami-Porta R. Transcervical videomediastino-thoracoscopy. J Thorac Dis 2018;10(Suppl 22):S2649-S2655. doi: 10.21037/ jtd.2018.03.132 